\title{
The Torino Observatory Parallax Program: White dwarf candidates
}

\author{
R. L. Smart, M. G. Lattanzi, B. Bucciarelli, G. Massone, R. Casalegno, G. Chiumiento, \\ R. Drimmel, L. Lanteri, F. Marocco, and A. Spagna
}

\begin{abstract}
Istituto Nazionale di Astrofisica (INAF), Osservatorio Astronomico di Torino, Strada Osservatorio 20, 10025 Pino Torinese, Italy
\end{abstract}

Received 21 January 2003 / Accepted 17 March 2003

\begin{abstract}
We present parallax determinations for six white dwarf candidates in the Torino Observatory Parallax Program. The absolute parallaxes are found with precisions at the 2-3 milliarcsec level. For WD 1126+185 we find a distance incompatible with being a white dwarf, implying an incorrect classification. For WD 2216+484 we find our distance is consistent with a simple DA white dwarf rather than a composite system as previously proposed in the literature. In general it is found that the published photometric distance is an overestimate of the distance found here.
\end{abstract}

Key words. white dwarfs - stars: distances - astrometry

\section{Introduction}

With the introduction of precise and efficient detectors such as the CCD and the Multichannel Astrometric Photometer (Gatewood 1987) the determination of parallaxes from the ground have undergone a revolution. There are now many programs that routinely find relative parallaxes with precisions of a few milliarcseconds (mas) see Dahn (1998) for a review. The largest of these programs is the USNO Flagstaff program (Monet et al. 1992, hereafter MON92) that uses a dedicated 1.5 reflector with a number of CCDs and routinely produces parallaxes with 1 mas precision. We have recently completed the Torino Observatory Parallax Program (TOPP) using an on site telescope specifically targeted at faint stars $\left(m_{v}>11\right)$ using the USNO program as a model. This program will complement the HIPPARCOS and USNO projects, producing comparable precision but for stars in the intervening magnitude range.

In this article we discuss the instrumentation, observational procedures, calibrations, reduction techniques and first results for six white dwarf candidates of the TOPP list.

\section{Instrument and observation procedures}

The 1.05m REOSC telescope of the Osservatorio Astronomico di Torino (OATo) is a long focus $(994.2 \mathrm{~cm}$ ) large plate scale $\left(20.7^{\prime \prime} / \mathrm{mm}\right)$ reflecting telescope with an astrometrically corrected field of view of $45^{\prime}$. The telescope is a scaled-down replica of the USNO $1.5 \mathrm{~m}$ telescope with a flat secondary mirror and a parabolic primary mirror. Observations are made

Send offprint requests to: R. Smart, e-mail: smart@to.astro.it using a large format $1296 \times 1152$ pixel EEV CCD05-30 with a per pixel scale of $00^{\prime \prime} 47$ and a field of view of $10^{\prime} \times 9^{\prime}$. All observations are carried out through a standard Cousins $I$ filter; this was done to reduce the effects of refraction but has the benefit of also being the zone of maximum quantum efficiency of the telescope/CCD system.

This telescope, filter and CCD combination was fixed, giving us a stable system which is required for astrometric studies of this nature. The TOPP was provided with $40 \%$ of the observational time on this telescope during the period 10 November 1994 to 2 July 2001. In Fig. 1 we plot the change in temperature, humidity, seeing and number of frames observed over the course of this period. In July 2001 the CCD was changed and the program for these targets was halted. The telescope is presently undergoing automation refitment and when that is stable we will begin another program with the new, higher resolution, CCD.

During the observational period the only major maintenance was a periodic realuminisation of the mirrors on 14/04/1997, 24/05/1999 and 04/12/2000. This required a dismounting of the mirrors and transportation to the Asiago Observatory. This procedure should not have an impact on the parallax program but we search for correlations with residuals in the solutions and these dates as a matter of routine.

For each target we make one $30 \mathrm{~s}$ exposure, then measure both the position and maximum pixel count for the target. The telescope is then repointed so that the target is in the center of the CCD and the exposure time is calculated in a simple manner to optimize the counts for the target and reference stars. In this way we insure that spatial variations on the CCD have minimal effect and that nightly variations of the sky are to a reasonable 

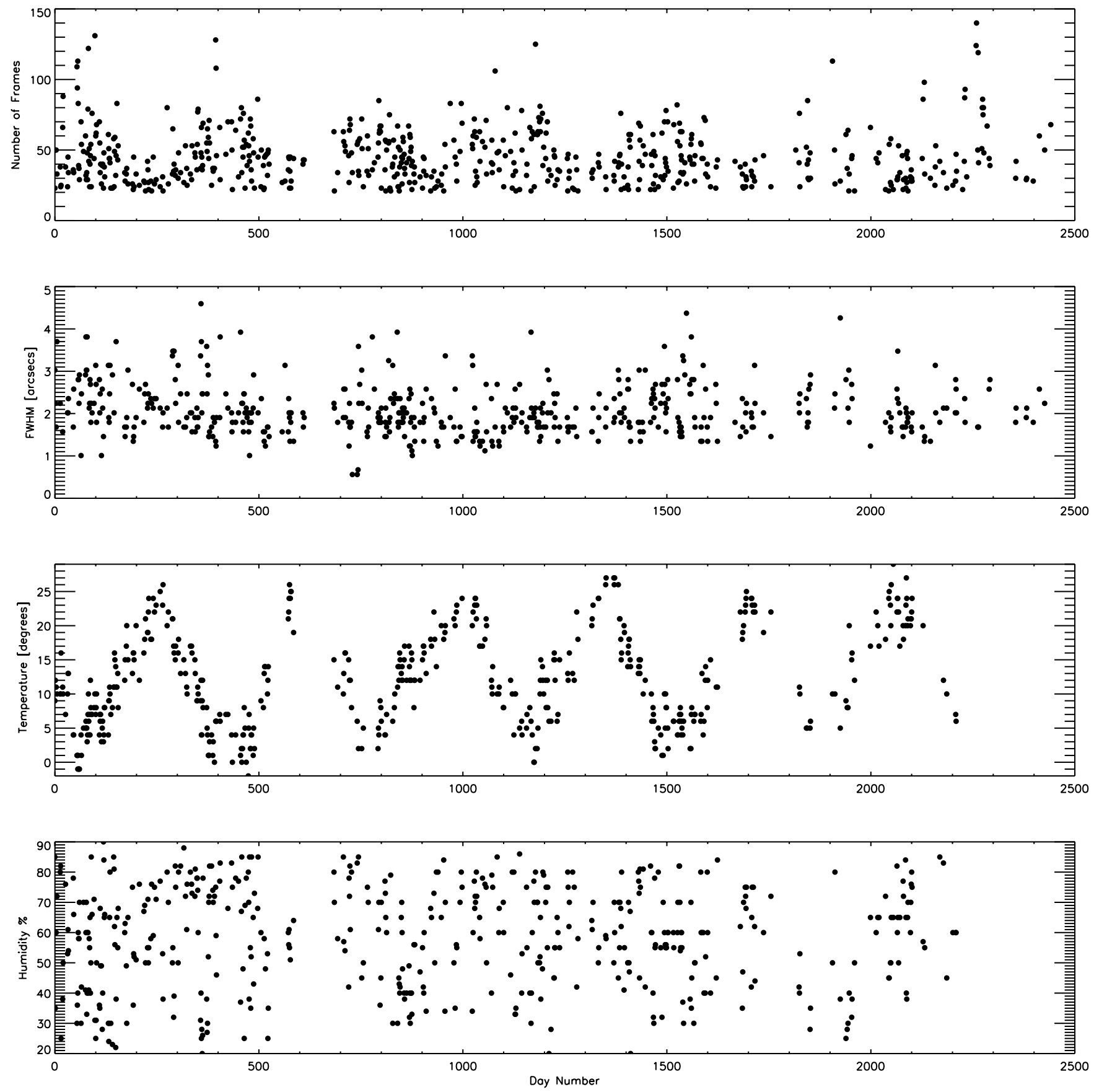

Fig. 1. The nightly number of frames taken, seeing, humidity and temperature conditions. Day number is from the 11 November 1994 , i.e. Julian date 2449667.0 .

extent accounted for. If the target is not too bright or faint and the reference stars not too sparse, then we choose a maximum pixel count that on a bad night will not require more than a five minute exposure and on a good night defaults to this optimal exposure time. We attempt to restrict all observations to within $30 \mathrm{~min}$ of the meridian and to make two exposures for each target per night.

\section{Calibrations}

The CCD images are processed using standard IRAF procedures. First all frames are bias subtracted using the overscan regions and then a flat is made for each night from a median of at least three sky flats. For each object image we flag pixels within $95 \%$ of the well limit and these are not used in the calculation of the centroids. Each image is divided by the median flat and then passed to an in-house program that automatically produces an object list using a simple three sigma above background criteria.

This object list and cleaned image is then processed to find the most important measured quantity in this project - the centroid. In Smart et al. (1999) we discuss the experiments carried out to determine the image centroids and conclude there that the best procedure is an in-house two-dimensional Gaussian 


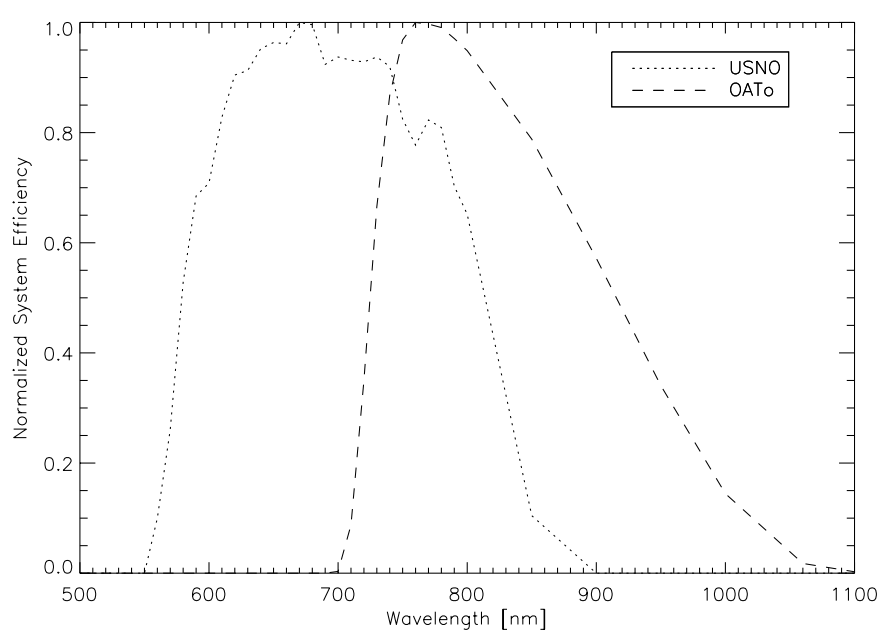

Fig. 2. The combined CCD quantum efficiency, optical transmission and filter transmission for the USNO Flagstaff $1.5 \mathrm{~m}$ reflector and the OATo $1.05 \mathrm{~m}$ reflector.

profile fitter. We estimate by comparison of consecutive frames that the centroiding precision is better than 0.02 pixels ( $\sim 10$ mas) for stars within 5 magnitudes of saturation and decreases rapidly for fainter stars.

The largest source of systematic error calibrated in MON92 was the effect of Differential Color Refraction (DCR). This occurs because the frame defined by the reference stars has a different average color than the target star. The position of the target star will be refracted differently than the mean field by the atmosphere and the size of this difference will be a function of the hour angle. This is especially important when observations were carried out far from the meridian to maximize the parallax factor.

In MON92 this effect was discussed at length and we are currently using data taken over the course of the program to calibrate the effect in a similar way. However we note that the combination of our CCD and longer wavelength filter means that our DCR will be much lower than theirs. In Fig. 2 we plot the combined CCD/filter/telescope efficency. In Smart et al. (1999) we show that the DCR of the Torino program is negligible in the case of QSOs which have similar colors to these white dwarfs and are close to the mean color of the reference stars. As a result of our low DCR, the restriction that observations be made close to the meridian, and a low color difference between the target and the mean reference star color, we believe we are justified in ignoring the DCR correction for these particular objects. Future work will include the DCR.

\section{Reduction techniques}

\subsection{Determination of relative parallax}

For the relative parallaxes found in this work we have used a simple iterative approach. We will eventually use a more rigorous procedure that will find directly the absolute parallax using a global central overlap procedure similar to that described in Eichhorn (1997). Since the stars under discussion here have parallaxes of tens of milliarcseconds we expect refinements such as the use of the central overlap to have minimal effect.

The selection of frames and reference stars is completely automatic. We have the ability to manually select the reference stars but testing on simulations has shown that an intelligent choice of selection criteria is sufficient and has the advantage of being free of observer bias. Initially all frames with an hour angle greater than one hour and all frames used to set up the target are dropped. A frame from the middle of the sequence with a high number of stars is chosen as the base-frame.

From the measured coordinates $(x, y)$ of objects on the base frame we calculate standard coordinates $(\xi, \eta)$ by a linear transformation using stars common to a reduction of the Digitized Sky Survey (DSS). This sets the plate scale and orientation which allows us to use parallax factors in standard coordinates. The precision of this transformation is limited by the quality of the DSS solution; for the orientation any errors less than $1 \mathrm{de}-$ gree do not affect our use of the standard coordinate parallax factors and we assume any errors in the scale are less than $1 \%$.

Using pattern recognition software we find 6 stars in common with the base frame and the other frames; any frame without at least six common stars is dropped. We then orient each frame to the standard coordinate system of the base frame using these 6 stars. Then we find all stars in common with the base frame and pick the 12 most frequent stars including the target star, all frames without these stars are dropped. These 12 stars define the reference system for the target star. Obviously if all the frames have a larger subset of stars in common then we will use that, but usually to have at least 12 stars we have to drop some of the frames.

We then find the frame parameters $A, B, C$ using the common stars by solving the transformation:

$\xi_{\mu, v_{\mathrm{o}}}=A_{v} x_{\mu, v}+B_{\nu} y_{\mu, v}+C_{v}$

where $\mu, v$ represent the star and frame number respectively and the $v_{\mathrm{o}}$ indicates the base plate. Here, and in further discussion, we develop the procedure in the $\xi$ coordinate but the relations are analogous in $\eta$. In calculating the parameters in Eq. (1) the target star is not used.

Then for all stars on the base frame we find a preliminary parallax and proper motion using:

$\xi_{\mu, v}=\xi_{\mu, v_{0}}\left(1.0+a_{\mu}\right)+\left(t_{v}-t_{v_{0}}\right) \mu_{\mu}+\left(P_{\xi_{v}}-P_{\xi_{v_{0}}}\right) \omega_{\mu}$.

Where: $a_{\mu}$ allows for the error in the base plate position, $\left(t_{v}-t_{v_{0}}\right)$ is the time difference, $\left(P_{\xi_{v}}-P_{\xi_{v_{0}}}\right)$ is the parallax factor difference and $\xi_{\mu, v_{0}}, \mu_{\mu}$ and $\omega_{\mu}$ are the position, proper motion and parallax of the star $\mu$. The parallax factors are given by: $P_{\xi}=\cos \alpha \sin \lambda \cos \epsilon-\sin \alpha \cos \lambda$ and $P_{\eta}=(\sin \epsilon \cos \delta-$ $\cos \epsilon \sin \alpha \sin \delta) \sin \lambda-\cos \alpha \sin \delta \cos \lambda$ where $\alpha, \delta$ are the fields equatorial coordinates, $\lambda$ is the solar longitude, and $\epsilon$ the inclination of the ecliptic.

From this fit we have predicted positions of the reference stars which we can then use to iterate again through Eqs. (1) and (2). Before doing this iteration we attempt to identify bad frames and stars by finding the root mean square of the residuals per frame and per star. Every frame or star with a root mean square greater than three sigma from the mean we delete from the next iteration. 
We iterate for at least 4 iterations and then stop when the change in the parallax is less than one mas. We attempted various forms of reweighting as we iterated both in the calculation of the plate parameters and in the calculation of the final parallax - however we found the best results by only applying weights in the calculation of the plate parameters in the final iteration. We have also reduced the sequences using Gaussfit (Jefferys et al. 1988), constraining the parallaxes and proper motions of the reference stars to zero, and this provide similar results, increasing our confidence in the solutions.

Using this reduction procedure we have implicitly assumed that the proper motions and parallaxes of the reference stars are uncorrelated within the frame. This will not be true for the proper motions, which in a given direction will be dominated by a combination of differential galactic rotation and solar motion, but this will only add a systematic error to the proper motions and we can ignore it as our main goal here is the parallax.

\subsection{Correction to an absolute parallax}

The quantity calculated above is the parallax of the target star relative to the anonymous reference stars. For each reference star we have also calculated a parallax and proper motion using the same reference system as that of the parallax star. Inevitably the reference star parameters $\left(a_{\mu}, \mu_{\mu}, \omega_{\mu}\right)$ are correlated with the definition of the reference system; however with at least 12 stars in each system we consider this correlation to be negligible.

From the target star's relative parallax we should subtract the mean parallax of the reference stars weighted by the weights used in the plate solutions and the dependences (Schlesinger 1911). This enforces the assumption that the reference frame is at a mean parallax of zero and the use of weights in this step allows us to consider each reference star as having unit weight in the final step for the correction from relative to absolute parallax. However, the dependences are all nearly equal and we expect this mean to be zero. We have found that usually the mean is much smaller than the variance of the various parallaxes and we have decided not to apply this correction as it is not sensibly different from zero.

As part of the calibration we observed standard magnitudes for all stars in each field. Using the Mendez \& van Altena (1996) galaxy model we can calculate the most probable distance of each reference star based on their magnitude. The mean of these distances is an estimate of the correction to absolute parallax that we must add to the calculated targets relative parallax. In Table 1 we list the range and mean of the reference star magnitudes and the corresponding relative to absolute correction (RAC) for each field.

\subsection{Determination of errors}

After the final iteration the formal error of the star parameters come directly from the final covariance matrix. As we used a nominal input for the centroiding error we multiply the terms of the covariance matrix by the error of unit weight.
Table 1. Magnitude ranges and means in the $I$ filter, and relative to absolute corrections in milliarcseconds as found from the Mendez \& van Altena galaxy model.

\begin{tabular}{ccccc}
\hline \hline Field & $I_{\min }$ & $I_{\max }$ & $\left.<I_{\mathrm{w}}\right\rangle$ & $\mathrm{RAC}$ \\
\hline $0322-019$ & 11.21 & 15.88 & 14.37 & 2.0 \\
$0423+120$ & 12.05 & 15.81 & 14.69 & 1.3 \\
$1126+185$ & 11.80 & 15.90 & 14.49 & 2.1 \\
$1716+020$ & 10.97 & 14.76 & 12.95 & 1.2 \\
$1840-111$ & 9.650 & 14.43 & 12.88 & 0.4 \\
$2216+484$ & 10.39 & 15.48 & 14.13 & 0.8 \\
\hline
\end{tabular}

Based on various considerations (see for example Smart et al. 1997) we estimate the error in the RAC found using a galaxy model to be about $30 \%$. Therefore to the formal relative parallax error above we add in quadrature $30 \%$ of the RAC to provide us with a final error.

We note that the use of the DSS to transfer the frame to the sky is basically a setting of the scale and orientation of the CCD. From an examination of the plate parameter errors and tests against external catalogs we have found that the scale of the DSS is correct to better than $1 \%$. This error is invisible to the calculation of the relative parallax and cannot be easily estimated on a case by case basis. We consider it a systematic error that for all of the targets here will be below the value of the formal error (being at most $1 \%$ of the targets parallax) and therefore if the user requires a maximum error estimate we suggest that they add $1 \%$ of the targets parallax to the quoted error.

The error on the proper motions is more difficult to access as it is affected directly by the orientation, scale, and the fact that they are relative as we have made no attempt to account for systematic motions of the reference stars. Any use of the proper motions quoted here that requires high precision should be done with care.

\section{Results}

In the TOPP program there are six white dwarf candidates for which we present the results here. These have been chosen because they will not be affected by the DCR which is currently not calibrated in the reduction procedure. In Table 2 we list the objects, number of reference stars $N_{\mathrm{s}}$, number of observations used in the final fit $N_{\mathrm{f}}$, absolute parallaxes $\omega$, proper motions $\mu_{\alpha}, \mu_{\delta}$, magnitude $V$, color $V-I$, the root mean square of the residuals between the observations and the final fit rms and the duration of the observational sequence in years $\Delta T$.

In Fig. 3 we present as a typical example the observed and fitted path for the target $0423+120$. The average residual is 12 and 10 milliarcsec in $\xi$ and $\eta$ respectively.

Two targets, $1716+020$ and 1840-111, are part of wide binary systems with M-dwarf stars. In both cases we find the difference in proper motions measured in these pairs are less than the errors. As can be seen from Table 3 both these systems have parallaxes in good agreement. The systematic direction of the existing difference, where the red star has larger parallax, is consistent with what is expected by not applying the DCR. 
Table 2. Parallaxes and proper motions for the six white dwarf candidates in the Torino Program. See text for explanation of columns.

\begin{tabular}{rrrcrcccrc}
\hline \hline WD Name & $N_{\mathrm{s}}$ & \multicolumn{1}{c}{$N_{\mathrm{f}}$} & \multicolumn{1}{c}{$\omega$ mas } & \multicolumn{1}{c}{$\mu_{\alpha}$ mas/yr } & \multicolumn{1}{c}{$\mu_{\delta}$ mas/yr } & $V$ & $V-I$ & rms mas & $\Delta T$ years \\
\hline $0322-019$ & 13 & 83 & $59.5 \pm 3.2$ & $222.5 \pm 1.0$ & $-857.5 \pm 1.2$ & $16.11 \pm 0.06$ & $0.87 \pm 0.11$ & 18.07 & 6.20 \\
$0423+120$ & 24 & 56 & $57.6 \pm 2.5$ & $-106.7 \pm 1.0$ & $-225.5 \pm 0.9$ & $15.43 \pm 0.04$ & $0.62 \pm 0.05$ & 10.94 & 5.31 \\
$1126+185$ & 11 & 41 & $0.9 \pm 2.4$ & $6.0 \pm 1.0$ & $7.2 \pm 1.1$ & $13.97 \pm 0.04$ & $0.75 \pm 0.05$ & 9.48 & 6.09 \\
$1716+020$ & 21 & 159 & $19.0 \pm 2.3$ & $-439.8 \pm 0.7$ & $-279.2 \pm 0.9$ & $14.30 \pm 0.10$ & $0.02 \pm 0.17$ & 18.49 & 6.27 \\
$1840-111$ & 77 & 64 & $34.9 \pm 5.1$ & $-234.7 \pm 1.6$ & $-247.7 \pm 1.5$ & $14.18 \pm 0.09$ & $0.38 \pm 0.14$ & 21.07 & 6.15 \\
$2216+484$ & 130 & 90 & $33.6 \pm 2.5$ & $146.7 \pm 1.2$ & $-40.3 \pm 0.8$ & $16.13 \pm 0.10$ & $0.85 \pm 0.15$ & 15.19 & 5.04 \\
\hline
\end{tabular}

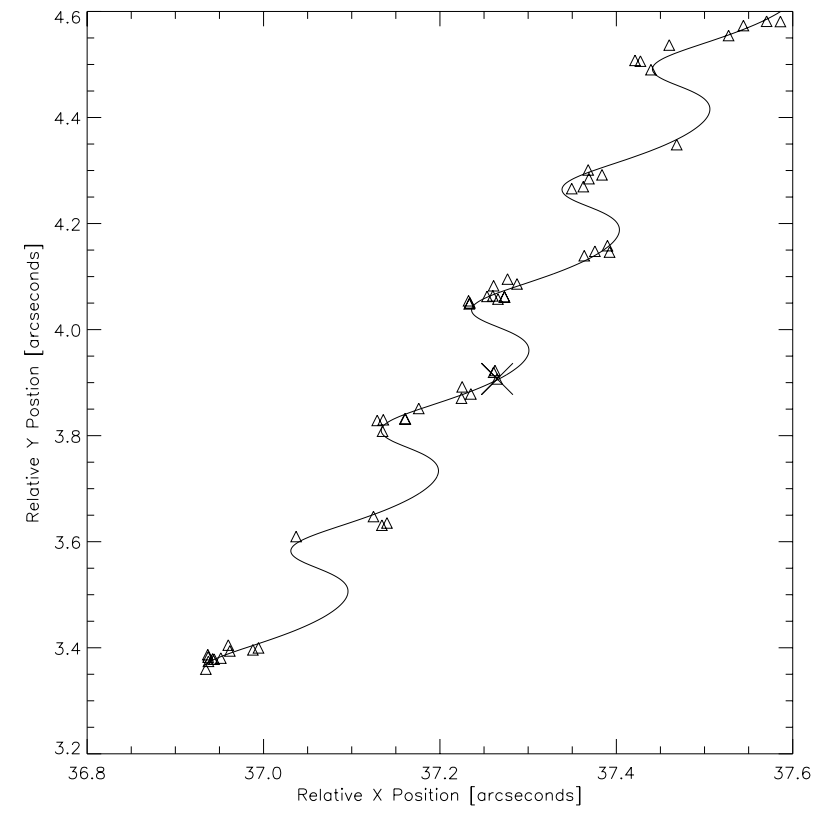

Fig. 3. Observations of $0423+120$ along with the fitted path. The $\mathrm{X}$ marks the position of the base plate.

Table 3. Parallaxes of two binary systems.

\begin{tabular}{rccr}
\hline \hline Target & $\omega$ mas & $V$ & $V-I$ \\
\hline $1840-111$ & $34.9 \pm 5.1$ & 14.18 & 0.38 \\
Companion & $37.9 \pm 4.0$ & 15.14 & 3.23 \\
$1716+020$ & $19.0 \pm 2.3$ & 14.30 & -0.02 \\
Companion & $21.6 \pm 1.3$ & 14.28 & 2.93 \\
\hline
\end{tabular}

Table 4. Observations from the Fourth Yale Parallax Catalogue for the determination of the parallax of $1716+020$.

\begin{tabular}{ccc}
\hline \hline Observatory & $1716+020$ & LHS3279 \\
\hline Lick & $24.4 \pm 0.9$ & $33.2 \pm 0.7$ \\
Sproul & $13.9 \pm 1.4$ & $45.9 \pm 1.6$ \\
USNO & $24.2 \pm 0.4$ & $31.4 \pm 0.4$ \\
OATo & $19.0 \pm 2.3$ & $21.6 \pm 1.3$ \\
\hline
\end{tabular}

Nevertheless the difference is of the order of the errors justifying our neglect of the DCR for these objects.

\subsection{Comments on individual targets}

0322-019 (LHS1547) is listed in the Astronomisches Rechen-Institut database for nearby stars (http://www.ari. uni-heidelberg.de/aricns/index.htm, hereafter ARICNS) as a DZ9 with a photometric parallax of 62 mas, consistent with our measurement of $59.5 \pm 3.2$.

$\mathbf{0 4 2 3 + 1 2 0}$ (EJ 169) is listed in the ARICNS as a DC8 with a photometric parallax of $81 \pm 11$ mas, significantly different from our value of 58 mas. This is derived from the absolute magnitude of McCook \& Sion (1999, hereafter MCC99) and the apparent magnitude in the ARICNS.

$\mathbf{1 1 2 6 + 1 8 5}$ (GJ 3667) is listed as a DC9 with a photometric parallax of $114 \pm 13$ mas, inconsistent with our zero value. We have used the finding chart provided in Green et al. (1986) where it was first identified as a composite DC white dwarf based on at least two spectra which were not published. Subsequent work with new spectroscopy by Putney (1997, Fig. 21) also confirmed this result with a spectra that indicated "a composite of a DC and a cool star". We have discussed these observations with various spectroscopists in light of this object's near zero proper motion and parallax, but have not been able to come up with other suggestions. As no other star in the TOPP field has a significant proper motion we therefore assume this is a case of misclassification and/or misidentification.

1716+020 (YPC 3926.01A, LHS3278) is listed in the Fourth Yale Parallax Catalog (van Altena et al. 2001) with a mean parallax of $28 \pm 2$ mas and a spectral type of DA6 which is inconsistent with our value of $19 \pm 2$ mas. However, as we noted before, this object has a companion red dwarf (LHS3279) and to calculate the Yale mean parallax the various observations of both stars were averaged. In Table 4 we list the individual observations. Considering $1716+020$ alone, our measurement is consistent with its mean parallax, whereas inconsistency arises when the parallax of the red dwarf companion is considered. This difference may be due to an incorrect treatment of the refraction effect in the other programs where it is much more important than ours. We also note that the projected distance in all cases is less than 0.016 parsecs while only our values can produce distances that are consistent with a line of sight difference of this order.

1840-111 (GJ 2139) is listed in the ARICNS as a DA5 with a photometric parallax of $53 \pm 6$ mas, inconsistent with our value of 35 mas. This is a derived value from the MCC 99 work and is discussed below. The higher error of this target may be a result of it's lower declination combined with the high field 
Table 5. Absolute magnitudes calculated from TOPP parallaxes and apparent magnitudes along with those derived in MCC99 from spectrophotometry, the code indicates the method used: $2=$ multichannel spectrophotometric colors, $3=u v b y$ colors.

\begin{tabular}{cccc}
\hline \hline Target & TOPP & MCC99 & code \\
\hline $0322-019$ & $14.98_{-.12}^{+.11}$ & 15.10 & 2 \\
$0423+120$ & $14.23_{-.10}^{+.09}$ & 15.05 & 3 \\
$1716+020$ & $10.69_{-.28}^{+.25}$ & 11.78 & 3 \\
$1840-111$ & $11.90_{-.34}^{+.30}$ & 12.81 & 3 \\
$2216+484$ & $13.76_{-.17}^{+.16}$ & 13.57 & 2 \\
\hline
\end{tabular}

density, which could adversely affect the centroiding by the crowding when the seeing was not optimal.

2216+484 (GD 402) has no parallax estimates in the literature. In Bergeron et al. (1990), based on anomalies in the spectra, they conclude that this object is probably a composite system of a DA and a DC white dwarf. From the apparent magnitude and our parallax we derive an absolute magnitude of 13.76 in $V$ which is consistent with a single DA star and contrary to the thesis of a double system which is estimated in Bergeron et al. to be of absolute magnitude 12.94. An examination of the point spread function during good seeing does not show any indication of duplicity. The apparent magnitude and the astrometric residuals of the system are stable over the program. These observations are not conclusive proof of singularity but they do put restrictions on orbit orientation in the case of a composite system.

\subsection{External comparisons}

In Table 5 we list the absolute magnitudes derived from the parallaxes and apparent magnitudes quoted here along with those derived in MCC99 from spectrophotometry. We have not included 1126+185 and we note that the 2216+484 MCC99 value is based on the assumption that it is a single DA7 star. It is beyond the scope of this paper to discuss the relative merits of absolute magnitudes derived from spectrophotometry versus those from parallaxes and the sample here is too small for such a comparison. However, there is a suggestion that the absolute magnitudes derived from $u v b y$ colors are underestimates (i.e. numerically larger) than the true magnitudes - assuming that there is not a systematic error of $30 \%$ in our results.

Apart from 1716+020 we do not have any direct comparisons to other trigonometric parallaxes determinations. We can however make an indirect comparison. In Fig. 4 we plot the white dwarf tract in the $\mathrm{H}-\mathrm{R}$ diagram as derived from both HIPPARCOS stars and those published in MON92 with the TOPP results over-plotted. We believe this adequately shows that our results are consistent with other high precision parallax determinations.

\section{Future work}

As we have stated the reduction technique used here is not optimal. The central overlap method developed by Eichhorn (1997)

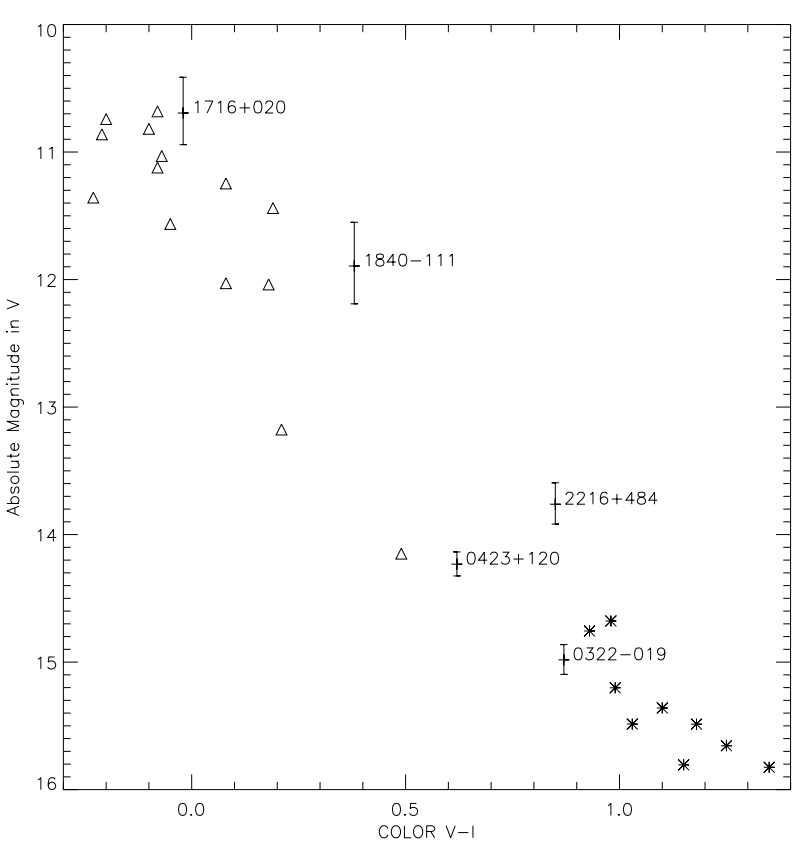

Fig. 4. The white dwarf sequence in the $\mathrm{H}-\mathrm{R}$ diagram. The asterisks are from MON92, triangles from HIPPARCOS and pluses with error bars this paper.

and extended by Rapaport (1998) is preferred. This method will allow the simultaneous adjustment of frame parameters, star parameters and the correction to the absolute frame using all the information available. It will mean that we will not have to use formal errors as weights, assume a mean parallax for the reference frame or neglect the dependencies. Significant external information is required for this procedure and it will probably only be important for stars which have parallaxes much closer to our precision limit where the relative error is larger. We do plan to implement this procedure in the future development of this program.

\section{Conclusion}

We have shown that the Torino program is able to produce useful parallaxes with precisions and in a magnitude range that complement the HIPPARCOS results and the USNO program. We plan to improve the reduction procedure which will only improve the precision and produce parallaxes for the other $100+$ targets observed over the last 6 years. Once the automatization of the REOSC telescope is complete we expect to be able to continue the program looking at the many exotic objects being discovered by the large all-sky surveys in progress.

We have measured the first parallax for five of six white dwarfs candidates. One object, $1126+185$, is obviously misclassified, another, $2216+484$, is inconsistent with previous observations that suggest it is a composite system. The overall photometric magnitude calibration of McCook \& Sion (1999) appears to overestimate the objects magnitude. These discrepancies with published work highlight the need for more direct parallax determinations which are fundamental tests of current theories. 
Note added in proof: Since going to press the ARICNS parallax for WD $0423+120$ has been updated with a new photometric parallax of 52 mas, consistent with the value derived here (Jahreiss personal communication).

Acknowledgements. First thanks go to the Consiglio Nazionale delle Ricerche for initially providing the CCD, and to the former Consiglio per la Ricerca Astronomica current INAF and the Directors of OATo for their longterm support of this program. A six year observational program could not have been carried out without the support of a number of the OATo staff. In particular we wish to thank Massimo Villata, Francesco Porcu, Fillipo Racioppi, Leonardo Corcione, Marco Del Bò, Francesco Salvati and the QSO monitoring group for observations made in service mode. Thanks to Bill Jefferys and Michel Rapaport for many useful discussions, also Dave Monet and Conrad Dahn for providing us with an initial kick start for this project. We would also like to thank the referee, Dr. van Leeuwen, whose comments helped improve the clarity of this paper. Finally, RLS is grateful to Heinrich Eichhorn who before his death was a constant source of guidance and through his work continues to inspire today. RLS acknowledges the past support of the Royal Society and Torino University.

\section{References}

Bergeron, P., Liebert, J., \& Greenstein, J. L. 1990, ApJ, 361, 190

Dahn, C. C. 1998, in Fundamental Stellar Properties, IAU Symp., 189, 19

Eichhorn, H. 1997, A\&A, 327, 404

Gatewood, G. 1987, AJ, 94, 213

Green, R. F., Schmidt, M., \& Liebert, J. 1986, ApJSS, 61, 305

Jefferys, W. H., Fitzpatrick, M. J., \& McArthur, B. E. 1988, Celestial Mechanics, 41, 39

McCook, G. P., \& Sion, E. M. 1999, ApJSS, 121, 1

Mendez, R. A., \& van Altena, W. F. 1996, AJ, 112, 655

Monet, D. G., Dahn, C. C., Vrba, F. J., et al. 1992, AJ, 103, 638

Putney, A. 1997, ApJSS, 112, 527

Rapaport, M. 1998, A\&A, 335, 769

Schlesinger, F. 1911, ApJ, 33, 161

Smart, R. L., Bucciarelli, B., Lattanzi, M. G., Massone, G., \& Chiumiento, G. 1999, A\&A, 348, 653

Smart, R. L., Lattanzi, M. G., \& Drimmel, R. 1997, in Wide-field Spectroscopy, ed. E. E. A. Kontizas (Kulwer Academic Press), 195

van Altena, W. F., Lee, J. T., \& Hoffleit, E. D. 2001, The General Catalogue of Trigonometric Stellar Parallaxes, Fourth edition (L. Davis Press) 